This is the post print version of the article, which has been published in Clinical Respiratory Journal 2019, vol 13, issue1, 34-42

Doi: https://doi.org/10.1111/cri.12977

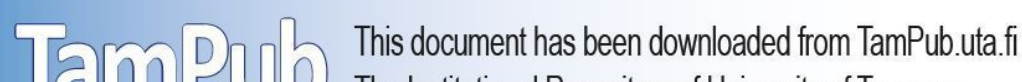 \\ The Institutional Repository of University of Tampere
}

\section{Implementation of a depression screening protocol among respiratory insufficiency patients}

Hanna Kerminen ${ }^{1,2}$, Esa Jämsen ${ }^{1,2}$, Pirkko Jäntti ${ }^{1}$, Aino K. Mattila ${ }^{3}$, Sirpa Leivo-Korpela ${ }^{4}$, and Jaakko Valvanne ${ }^{1}$

1 University of Tampere, Faculty of Medicine and Life Sciences, and the Gerontology Research Centre (GEREC), Tampere, Finland

2 Tampere University Hospital, Centre of Geriatrics, Tampere, Finland

3 Tampere University Hospital, General Hospital Psychiatry Unit, Department of Adult Psychiatry, Tampere, Finland

4 Tampere University Hospital, Department of Respiratory Diseases, Tampere, Finland

Corresponding author: Hanna Kerminen

Postal address: PL 100, 33014 Tampereen yliopisto, Finland

e-mail address: hanna.kerminen@uta.fi

tel: +358405327042

Running title: Implementation of a depression screening protocol

An authorship statement:

Hanna Kerminen designed a depression screening protocol and evaluation with other authors, collected and analysed data and was the first writer of the manuscript. Esa Jämsen participated in analysing data and writing the manuscript. Pirkko Jäntti, Aino K. Mattila, Sirpa Leivo-Korpela and Jaakko Valvanne participated in designing a depression screening protocol and evaluation and analysing data. They also took part in writing process.

Disclosure statement:

The authors of this publication (Hanna Kerminen, Esa Jämsen, Pirkko Jäntti, Aino K. Mattila, Sirpa LeivoKorpela and Jaakko Valvanne) declare that they have no significant competing financial, professional, or personal interests that might have influenced the performance or presentation of the work described in this manuscript. 


\section{Abstract}

Introduction. Unnoticed and untreated depression is prevalent among patients with chronic respiratory insufficiency. Comorbid depression causes suffering and worsens patients' outcomes.

Objectives. The objective of this evaluation was to assess preliminary outcomes of a depression screening protocol among chronic respiratory insufficiency patients at a tertiary care pulmonary outpatient clinic.

Methods. In the depression screening protocol, the patients filled the Depression Scale (DEPS) questionnaire. Patients whose scores suggested depression were offered the opportunity of a further evaluation of mood at a psychiatric outpatient clinic. The outcomes of the protocol were evaluated retrospectively from the patient records.

Results. During the period of evaluation, 238 patients visited the outpatient clinic. DEPS was administered to 176 patients (74\%), of whom 60 (34\%) scored $\geq 9$ (out of 30), thus exceeding the cut-off for referral. However, only 13 patients were referred, as the remainder declined the referral. Finally, seven patients were evaluated at the psychiatric clinic, and they all were deemed depressive. Symptoms of depression were most prevalent among patients with a long smoking history, refractory dyspnoea, and a history of depression.

Conclusion. Depression screening was positive in a third of the patients. The depression screening protocol improved the detection of depression symptoms, but the effects on the patients' treatment and clinical course were small. Rather than referring patients to a psychiatric unit, the evaluation and management of depression should be undertaken at the pulmonary unit.

Key words: respiratory insufficiency, depression, health services research 


\section{Introduction}

Depression is a common comorbidity in severe chronic pulmonary diseases. Depression diminishes functional performance and exercise tolerance while increasing fatigue, hospital admissions, morbidity and mortality $(1,2)$. The risk for developing depression increases with the severity of chronic obstructive pulmonary disease (COPD) (3), and up to $30-50 \%$ of patients with severe COPD have depression $(4,5)$. In patients with oxygen-dependent chronic respiratory insufficiency, the prevalence of depression may be as high as $60-75 \%(6,7)$.

Depression is often undetected and untreated among respiratory insufficiency patients (8). According to previous reports, less than a third of COPD patients suffering from depression are being treated for it (9-11). There are several possible reasons for the low detection rate. Firstly, the diagnosis of depression is challenging, because the symptoms of pulmonary disease may resemble those of depression. In addition, both patients and health care personnel may consider psychiatric symptoms a normal reaction to having progressive illness rather than suspecting comorbid psychiatric disease (12). Finally, depression is rarely screened for in routine health care.

As comorbid depression is widely undetected, routine screening for depression has been recommended $(13,14)$. Nevertheless, screening should only be performed if a local depression treatment pathway with the possibility of consulting a psychiatrist has been established (13). Thus, it is essential to implement not only the screening instrument but also a care pathway allowing appropriate diagnostics and treatment for patients with positive screening results. However, the implementation of new protocols in clinical practice is usually challenging, and a variety of problems may arise at different organisational levels $(15,16)$.

In this paper, we describe the implementation and preliminary results of a depression screening protocol among respiratory insufficiency patients at a pulmonary outpatient clinic. To the best of our knowledge, such care pathways have not been described previously.

\section{Materials and Methods}

\section{Setting}

Tampere University Hospital is a tertiary hospital situated in the southern part of Finland. It provides specialised care to about 530,000 people living in 23 municipalities situated within a $110 \mathrm{~km}$ radius from the city of Tampere. Patients with respiratory diseases are treated at the outpatient clinic; the annual volume is about 14,000 visits by 4,500 patients. At the outpatient clinic, there is a specialised section for respiratory insufficiency patients; its annual volume is approximately 500 patients.

The patients are referred to the respiratory insufficiency section by physicians working in primary, private, or specialised health care. Typically, the patients have a severe lung, heart, or neurological disease with suspected chronic respiratory insufficiency. At the clinic, a pulmonologist meets the patient, considers the differential diagnosis, and makes decisions about medications and possible 
device treatments (long-term oxygen therapy (LTOT) and/or non-invasive ventilation (NIV)). A multidisciplinary team consisting of a nurse, a physiotherapist, a social worker, a dietician, and a rehabilitation counsellor participates in the care. Follow-up is organised according to a discrete protocol.

\section{Implementation of the depression screening protocol}

When interviewing the patients at the outpatient clinic, nurses have noticed that the patients often have depressive symptoms but no treatment for depression. The nurses also noted their lack of knowledge of how to approach such symptoms, especially as patients with chronic respiratory insufficiency often have difficulties in seeking help because of restricted functional ability.

From this background, we decided to start the development and implementation of a depression screening protocol. The protocol was developed in collaboration with the general hospital psychiatric unit. For this project, a contract was drawn up to allow the referral of screeningpositive patients to the psychiatric outpatient clinic even though the usual referral criteria were not met. The personnel of the pulmonary unit were educated about depression detection by lectures and group discussions. The aim was to improve the identification of depressive symptoms, bring up mood symptoms in discussion, and enhance their further evaluation and treatment.

The Depression Scale (DEPS) was selected as the screening instrument. The DEPS is a validated, self-rated screening tool for depression (17). It is the primary screening instrument for depression at Tampere University Hospital. The DEPS questionnaire consists of ten items, and scores vary from 1 to 30 points. The cut-off point for depressive symptoms is $\geq 9$, while the cut-off point for clinical depression is $\geq 12$ (18).

Screening commenced in the autumn of 2015. Nurses were instructed to administer the DEPS questionnaire to every patient visiting the respiratory insufficiency section. A referral to an appointment at the psychiatric outpatient clinic was offered to patients with a positive screening. A pulmonologist made the referral, and the patient was later informed of the appointment time. In 2015 , the cut-off for referral was $\geq 12 / 30$ points. In 2016, the cut-off was lowered to $\geq 9 / 30$ to include patients with milder symptoms. According to Sheehan and McGee (2013), a lower cut-off score increases the possibility of identifying depression (greater sensitivity), whereas a higher cutoff score diminishes false-positive results (greater specificity) at the cost of sensitivity (19).

\section{Evaluation of screening}

Evaluation of the screening protocol was made retrospectively from the patient records. The patients included in the study were those who visited the respiratory insufficiency section during three different time periods: 17 Aug - 23 Oct 2015 (pilot phase I), 9 Nov 2015 - 15 Jan 2016 (pilot phase II), and 15 Sep - 31 Dec 2016 (follow-up phase).

Patient records were reviewed to evaluate: 1 ) the coverage of the screening; 2 ) the patients' willingness to fill the DEPS questionnaire; 3 ) the proportion of patients with positive DEPS scores; 4) the patient characteristics associated with high DEPS scores; and 5) the consequences of positive screenings. 
To identify patients at an elevated risk for depression, we registered each patient's age, gender, use of walking aids and home care, living arrangements, smoking history, pulmonary disease diagnosis, causes for chronic respiratory insufficiency, other diagnoses, use of psychoactive medications, available measurements of lung function ( $\mathrm{FEV}_{1}$ in post-bronchodilator spirometry), and functional exercise capacity (6-Minute Walk test, 6MWT). In addition, the measurements made during the visit were gathered, including the patient's height, weight, microspirometry, and the scores of the Alcohol Use Disorders Identification Test (AUDIT-C) (20), the modified Medical Research Council Dyspnoea Scale (mMRC) (21), and the COPD Assessment Test (CAT) (22). Spirometry was performed using the Vmax 20 spirometer (Sensor-Medics, Yorda Linda, CA, USA) and microspirometry was performed using a microspirometer (Vitalograph ${ }^{\circledR} \operatorname{copd}-6^{\mathrm{TM}}$, Vitalograph, Ennis, Ireland).

\section{Statistics}

The process of the depression screening protocol is reported descriptively. To identify patient groups with a high prevalence of depression symptoms, the associations of the above-mentioned patient characteristics with DEPS scores $<9$ vs $\geq 9$ and $<12$ vs $\geq 12$ were analysed. In addition, the results of microspirometry, spirometry, 6MWT, CAT, MMRC, and AUDIT-C were compared with the DEPS scores.

Statistical significance between groups was analysed using the Mann-Whitney $U$ test, the chi-squared test, or Fisher's test as appropriate. A $p$-value $<0.05$ was considered statistically significant. Data management and analysis were performed using IBM SPSS Statistics 23.

\section{Ethics}

The implementation of depression screening in routine care was part of development work at the pulmonary outpatient clinic. The patients could refuse to fill the DEPS questionnaire. The retrospective study was organised to evaluate the outcomes of the screening, and the patients were not approached by the researchers. Prior to commencing the study, permission was acquired from the Science Centre of Tampere University Hospital.

\section{Results}

\section{Baseline characteristics of the patients}

In total, 242 patients visited the respiratory insufficiency section during the defined time periods. Four patients using ventilators were excluded, leaving 238 for evaluation. Table 1 illustrates the characteristics of the included patients. Of the patients, $38(16 \%)$ were attending their first visit, while the remainder had made earlier visits. A third of the patients attended a nurse's visit only; the rest met both a nurse and a physician.

Most patients had a diagnosis of chronic respiratory insufficiency $(n=200,84 \%)$, and in $75 \%$ $(n=150)$ the diagnosis had been made less than five years ago. Nearly half of these patients $(n=82)$ were considered to have more than one disease diagnosis as the cause for their respiratory insufficiency. The most common diagnoses were COPD ( $n=105,53 \%)$, obstructive sleep apnoea 
( $n=66,33 \%)$, and obesity hypoventilation $(n=54,27 \%)$. Other diagnoses related to respiratory insufficiency were pulmonary fibrosis ( $n=17,9 \%)$, pulmonary hypertension $(n=17,9 \%)$, neurological disorders $(n=14,7 \%)$, deformities of the chest wall $(n=9,5 \%)$, elevated hemidiaphragm $(n=7,4 \%)$, and miscellaneous causes $(n=11,6 \%)$. Hypertension $(n=134,56 \%)$, type 2 diabetes $(n=80,34 \%)$, and coronary artery disease $(n=46,23 \%)$ were the most common comorbidities. Forty-five patients (19\%) had a previous diagnosis of depression, 16 (7\%) had another psychiatric diagnosis, and 17 (7\%) had a memory disorder. Thirty-eight patients (16\%) were using antidepressants, 88 (37\%) were using anxiolytic drugs, and 23 (10\%) were using antipsychotics.

\section{Outcomes of the screening}

The DEPS questionnaire was filled by $74 \%$ of the patients $(n=176)$. The proportion of the patients that completed the DEPS questionnaire increased from $66 \%$ in the first year to $88 \%$ in the second year. Only six patients refused to fill the DEPS questionnaire. The unscreened patients were younger and had a lung disease diagnosis other than COPD more often compared to the screened patients.

Depression screening was positive in a quarter to a third of the patients, depending on the cut-off point (Figure 1). Referral to psychiatric services was offered to most patients with a positive screening. However, more than three quarters of them declined the referral. The reasons for declining the referral were not systematically recorded, but there were a few notes relating to difficulties with travelling, and indeed, 19 of the 24 patients who declined the referral lived outside the city of Tampere where the psychiatric clinic is situated.

Altogether, 13 referrals were made. Seven patients met a nurse or a psychologist at the psychiatric outpatient clinic. Of the remaining patients, four could not travel to the clinic; instead, they received a phone call to assess the severity of symptoms. Two patients died before the time of the appointment. All patients visiting the clinic were deemed depressive. After one or two visits, all patients were directed to further care at regional psychiatric services.

Identifying the patients with a positive depression screening

Table 2 illustrates the associations of the DEPS scores with the patients' characteristics. High DEPS scores were common in patients who used walking aids or had a history of depression or heavy smoking. Depression screening was positive in $44 \%$ of the COPD patients, $38 \%$ of the sleep apnoea patients, and $29 \%$ of the obesity hypoventilation syndrome patients. Depression screening was positive in $43 \%$ of the patients using LTOT and $33 \%$ of the patients using NIV. Screening was positive in $58 \%$ of the patients with a history of depression and in $44 \%$ of the patients using antidepressants.

Table 3 illustrates the associations of the DEPS scores with the measurements of lung function and assessment tests. The patients with high DEPS scores also had high scores in the CAT and mMRC tests. The FEV 1 in spirometry and in microspirometry, AUDIT-C scores and distance in 6MWT were not associated with the DEPS scores. 


\section{Discussion}

\section{Symptoms of depression}

Supporting earlier observations $(4,6,7)$, symptoms of depression were prevalent in patients: depression screening was positive in a third of patients. In line with earlier observations $(3,9)$, only a minority had a prior diagnosis of depression or were using antidepressants, underlining the need for depression screening. Prior depression, a long smoking history, and the use of walking aids were associated with having symptoms of depression. In addition, symptomatic COPD patients with functional limitations owing to dyspnoea often experienced depression symptoms. Daily smoking has been shown to be a risk factor for depression (22), and prior depression predisposes to new depression (23). A relationship between CAT scores $>20$ and depression has been reported previously $(24,25)$. Furthermore, the perception of dyspnoea is related to psychological factors, meaning that symptoms of depression increase dyspnoea (26). Therefore, high CAT or mMRC scores should be taken as sign warranting broader evaluation of the patient beyond lung disease-specific aims (27).

\section{Implementation of depression screening}

The challenges that we met when implementing the screening protocol can be divided into three groups based on the integrated checklist of determinants of practice (the TICD checklist) (28): individual health professional factors, patient factors, and professional interactions.

Individual health professional factors include knowledge and skills, attitudes, and professional behaviour (28). The acceptability of the screening among nurses, measured as the proportion of patients who received the DEPS questionnaire, was not good in the first year of screening, but it improved in the second year. Nevertheless, one in ten patients went unscreened in the second year, although in itself, the inclusion of the DEPS questionnaire in the clinic visit appeared feasible. The nurses felt comfortable asking the patients to fill the DEPS questionnaire, but they experienced difficulties regarding how to discuss positive screening results with the patients. Future education of the healthcare professionals at the pulmonary clinic should therefore focus on communication and supportive discussion with a depressed patient. Emphasizing the importance of separating the symptoms of depression from those of pulmonary disease - and seeing improvement in both symptoms and quality of life when mood disorder is treated - would help in motivating for systematic, continuous use of the screening tool.

Patient factors include beliefs and knowledge, motivation, and behaviour (28). The acceptability of completing the DEPS questionnaire was good, but the acceptability of referral for further evaluation was not: most of the screening-positive patients declined referral. This may partly be explained by geographical obstacles, but there are other possible explanations. Generally, COPD patients tend to deny depressive symptoms and usually refuse to accept referral to psychiatric services $(12,29)$. The fear of stigmatisation concerning a psychiatric diagnosis may be one reason. Moreover, being unaware of the symptoms of depression, many patients may think that feeling depressed is a normal reaction to having a progressive somatic illness (8). Therefore, after a positive depression screening, it is important to educate patients about depression and to explain 
the potential advantages of seeking help (12). More than one discussion may be needed to achieve this.

Professional interactions include communication, team processes, and referral processes (28). We failed to equip the professional teams with adequate skills and resources. Implementing a new protocol in clinical practice successfully requires changes in diverse levels of care and time for adjustments. In particular, the nurses at the pulmonary clinic lacked time and knowledge, and there were insufficient resources to have a psychiatric nurse attend the pulmonary outpatient clinic to interview the patients there. In addition, local care pathways for depression in surrounding communities were not involved in the protocol. In conclusion, the capacity for organisational change was not sufficient to manage patients with a positive screening.

After the implementation of the screening, the detection of depressive symptoms certainly improved, but it is unclear how the screening affected the patients' clinical course. Optimally, the detection and treatment of depression would improve quality of life and also reduce the pulmonary disease symptoms (30). Therefore, screening for depression is recommendable, but the issue to be resolved is how to organise services so that they are both accessible and acceptable from the patients' point of view.

In the future, greater efforts are needed to ensure that appropriate discussion is available after a positive screening and further evaluation of mood is more accessible for the patients. Taken together, one solution would be to conduct a further evaluation of mood at the pulmonary clinic alongside screening instead of referring patients to a psychiatric unit. For the patients, the pulmonary unit is a natural environment to deal with the comorbidities of the pulmonary disease. The fear of stigmatisation related to the diagnosis of depression would likely be lower in pulmonary than psychiatric unit. Furthermore, such approach would reduce the need for separate hospital visits that would be burdensome to the patients. The problems to be solved include clarifying who is competent to perform the evaluation and how the resources should be guided so that the patients receive a timely evaluation. However, the best solution for the patients would probably be a care pathway that is connected to local health care, enabling the patients to receive evaluation and treatment (when necessary) close to their homes. This is challenging, however, because practices and the availability of services concerning the suspicion of depression vary greatly between municipalities.

\section{Limitations}

This evaluation was part of developmental work at the pulmonary outpatient clinic. These data must be interpreted with caution because our study was a retrospective evaluation of depression screening outcomes. One weakness was that neither the patients nor the nurses were systematically interviewed for the study; thus, not all possible contributing factors were fully clarified. Our initial purpose was not to search for patient groups with an elevated risk for depression, but some risk groups were nevertheless identified in the evaluation. 


\section{Conclusion}

Unnoticed symptoms of depression are prevalent among patients with chronic respiratory insufficiency. After commencing a protocol for depression screening in the pulmonary outpatient clinic, the detection of depression symptoms improved, but the effects on the patients' clinical course were small. The patients' compliance with the further evaluation of mood was poor. Screening for depression is recommendable, but the further assessment of patients with a positive screening should be organised in a way that is more acceptable and achievable from the patient's point of view. Instead of referring patients to a psychiatric unit, the evaluation and management of depression should rather be performed in the pulmonary unit.

\section{References}

(1) Norwood R. Prevalence and impact of depression in chronic obstructive pulmonary disease patients. Curr Opin Pulm Med 2006; 12: 113-117.

(2) Pooler A, Beech R. Examining the relationship between anxiety and depression and exacerbations of COPD which result in hospital admission: a systematic review. Int J Chron Obstruct Pulmon Dis 2014; 9: 315330.

(3) Kim KU, Park HK, Jung HY, Ahn JJ, Moon E, Kim YS, et al. Association of depression with disease severity in patients with chronic obstructive pulmonary disease. Lung 2014; 192: 243-249.

(4) Janssen DJ, Spruit MA, Leue C, Gijsen C, Hameleers H, Schols JM, et al. Symptoms of anxiety and depression in COPD patients entering pulmonary rehabilitation. Chron Respir Dis 2010; 7: 147-157.

(5) Stage KB, Middelboe T, Pisinger C. Measurement of depression in patients with chronic obstructive pulmonary disease (COPD). Nord J Psychiatry 2003; 57: 297-301.

(6) Kayhan F, llik F, Karamanli H, Pazarli AC, Kayhan A. Major depression in long-term oxygen therapy dependent chronic obstructive pulmonary disease. Perspect Psychiatr C 2016; 54: 6-10.

(7) Lacasse $Y$, Rousseau L, Maltais F. Prevalence of depressive symptoms and depression in patients with severe oxygen-dependent chronic obstructive pulmonary disease. J Cardiopulm Rehabil 2001; 21: 80-86.

(8) Ouellette DR, Lavoie K. Recognition, diagnosis, and treatment of cognitive and psychiatric disorders in patients with COPD. Int J Chron Obstruct Pulmon Dis 2017; 12: 639-650.

(9) Kunik ME, Roundy K, Veazey C, Souchek J, Richardson P, Wray NP, et al. Surprisingly high prevalence of anxiety and depression in chronic breathing disorders. Chest 2005; 127: 1205-1211.

(10) Jordan N, Lee T, Valenstein M, Weiss K. Effect of care setting on evidence-based depression treatment for veterans with COPD and comorbid depression. J Gen Intern Med 2007; 22: 1447-1452.

(11) Wong T, Xiang Y, Tsoh J, Ungvari GS, Ko FWS, Hui DSC, et al. Depressive disorders in older patients with chronic obstructive pulmonary disease (COPD) in Hong Kong: A controlled study. Aging Ment Health 2014; 18: 588-592. 
(12) Yohannes AM, Baldwin RC, Connolly MJ. Depression and anxiety in elderly patients with chronic obstructive pulmonary disease. Age Ageing 2006; 35: 457-459.

(13) Depression. Current Care Guidelines. Working group set up by the Finnish Medical Society Duodecim and the Finnish Psychiatric Association. Helsinki: The Finnish Medical Society Duodecim, 2016 (referred March 3, 2018). Available online at: www.kaypahoito.fi.

(14) Nici L, Donner C, Wouters E, Zuwallack R, Ambrosino N, Bourbeau J, et al. American Thoracic Society/European Respiratory Society statement on pulmonary rehabilitation. Am J Respir Crit Care Med 2006; 173: 1390-1413.

(15) Peters DH, Adam T, Alonge O, Agyepong IA, Tran N. Implementation research: what it is and how to do it. BMJ 2013; 347: f6753.

(16) Brommels M. Management and medicine: odd couple no more. Bonding through medical management research. Scand J Public Health 2010; 38: 673-677.

(17) Salokangas RK, Poutanen O, Stengard E. Screening for depression in primary care. Development and validation of the Depression Scale, a screening instrument for depression. Acta Psychiatr Scand 1995; 92: 10-16.

(18) Poutanen O, Koivisto AM, Kaaria S, Salokangas RK. The validity of the Depression Scale (DEPS) to assess the severity of depression in primary care patients. Fam Pract 2010; 27: 527-534.

(19) Sheehan AM, McGee H. Screening for depression in medical research: ethical challenges and recommendations. BMC Med Ethics 2013; 14: 4-7.

(20) Bush K, Kivlahan DR, McDonell MB, Fihn SD, Bradley KA. The AUDIT alcohol consumption questions (AUDIT-C): an effective brief screening test for problem drinking. Arch Intern Med 1998; 158: 1789-1795.

(21) Mahler DA, Wells CK. Evaluation of Clinical Methods for Rating Dyspnea. Chest 1988; 93: 580-586.

(22) Pasco JA, Williams L, Jacka FN, Ng F, Henry MJ, Nicholson GC, et al. Tobacco smoking as a risk factor for major depressive disorder: population-based study. Br J Psychiatry 2008; 193: 322-326.

(23) Cole MG, Dendukuri N. Risk factors for depression among elderly community subjects: a systematic review and meta-analysis. Am J Psychiatr 2003; 160: 1147-1156.

(24) Silva Júnior JL, Conde MB, de Sousa Corrêa K, da Silva C, da Silva Prestes L, Rabahi MF. COPD Assessment Test (CAT) score as a predictor of major depression among subjects with chronic obstructive pulmonary disease and mild hypoxemia: a case-control study. BMC Pulm Med 2014; 14: 186-192.

(25) Lee YS, Park S, Oh Y, Lee S, Park S, Kim YS, et al. Chronic obstructive pulmonary disease assessment test can predict depression: a prospective multi-center study. J Korean Med Sci 2013; 28: 1048-1054.

(26) von Leupoldt A, Dahme B. Psychological aspects in the perception of dyspnea in obstructive pulmonary diseases. Respir Med 2007; 101: 411-422.

(27) Masaki M, Hidetoshi N, Shotaro C, Mamoru S, Mizuha H, Shuichi Yoshida, et al. Analysis of comorbid factors that increase the COPD assessment test scores. Resp Res 2014; 15: 13-20. 
(28) Flottorp SA, Oxman AD, Krause J, Musila NR, Wensing M, Godycki-Cwirko M, et al. A checklist for identifying determinants of practice: a systematic review and synthesis of frameworks and taxonomies of factors that prevent or enable improvements in healthcare professional practice. Implement Sci 2013; 8: 35-45.

(29) Maurer J. Anxiety and depression in COPD: current understanding, unanswered questions, and research needs. Chest 2008; 134: 43-56.

(30) Momtaz OM, Rabei SM, Tawfike NR, Hasan AA. Effect of treatment of depression and anxiety on physiological state of severe COPD patients. Egypt J Chest Dis Tuberc 2015; 64: 29-34.

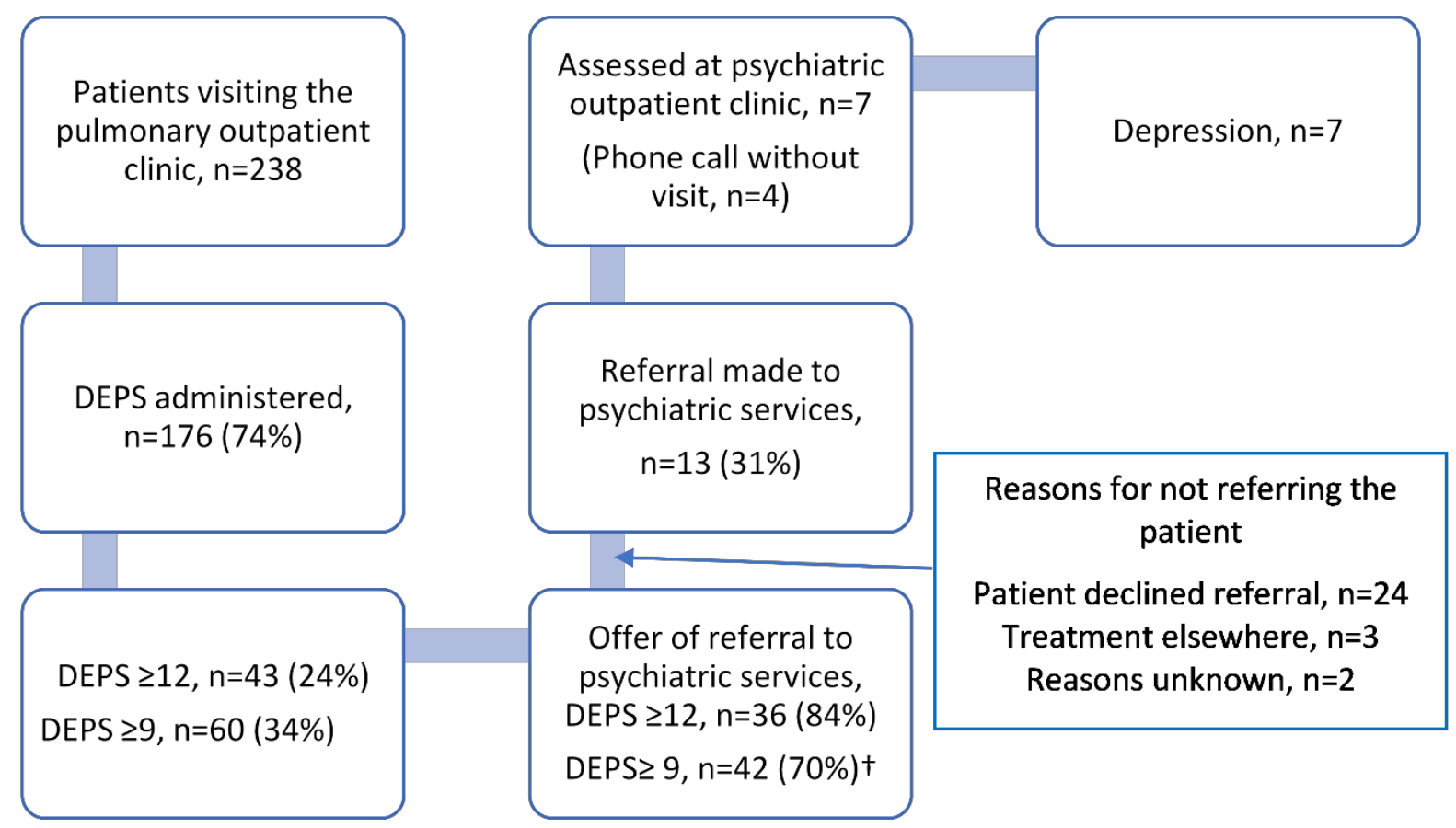

TDEPS 9-11 points in 2015, referral not instructed, $n=8$.

Figure 1. Realisation of the depression screening in the pulmonary outpatient clinic's respiratory insufficiency section and referral to further evaluation at the psychiatric outpatient clinic. 
Table 1. Baseline characteristics of the patients.

\begin{tabular}{|c|c|c|}
\hline & $\mathrm{n}$ & $\%$ \\
\hline \multicolumn{3}{|l|}{ Gender } \\
\hline Male & 138 & 58 \\
\hline Female & 100 & 42 \\
\hline \multicolumn{3}{|l|}{ Age (years) } \\
\hline$<70$ & 86 & 36 \\
\hline $70-80$ & 95 & 40 \\
\hline$>80$ & 57 & 24 \\
\hline \multicolumn{3}{|l|}{ Place of domicile } \\
\hline Tampere & 95 & 40 \\
\hline another municipality in the Pirkanmaa region & 143 & 60 \\
\hline \multicolumn{3}{|l|}{ Residence } \\
\hline own home & 216 & 91 \\
\hline sheltered housing & 12 & 5 \\
\hline nursing home & 10 & 4 \\
\hline \multicolumn{3}{|l|}{ Home care or assistance } \\
\hline none & 164 & 69 \\
\hline communal home care & 38 & 16 \\
\hline close relative as a carer & 17 & 7 \\
\hline nursing home staff & 10 & 4 \\
\hline personal assistant & 9 & 4 \\
\hline Use of walking aids & 131 & 45 \\
\hline Current smoking & 19 & 8 \\
\hline \multicolumn{3}{|l|}{ Smoking history in pack-years } \\
\hline no smoking history & 75 & 32 \\
\hline$<20$ & 31 & 13 \\
\hline $20-40$ & 70 & 29 \\
\hline$>40$ & 62 & 26 \\
\hline \multicolumn{3}{|l|}{ Use of long-term oxygen therapy or non-invasive ventilation } \\
\hline long-term oxygen therapy & 88 & 37 \\
\hline portable oxygen therapy & 75 & 32 \\
\hline bilevel positive airway pressure (NIV) & 106 & 45 \\
\hline continuous positive airway pressure (CPAP) & 17 & 7 \\
\hline \multicolumn{3}{|l|}{ Body mass index $(\mathrm{BMI}) \mathrm{kg} / \mathrm{m}^{2}(\mathrm{n}=227)$} \\
\hline underweight $<18.5$ & 12 & 5 \\
\hline normal 18.5-24.9 & 69 & 30 \\
\hline overweight $25-29.9$ & 41 & 18 \\
\hline obese $\geq 30$ & 101 & 45 \\
\hline
\end{tabular}


Table 2. Association of the DEPS scores with the patients' baseline characteristics in two groups with different cut-off points (DEPS $<9$ vs $\geq 9$ and DEPS $<12$ vs $\geq 12$ ).

\begin{tabular}{|c|c|c|c|c|c|}
\hline & & DEPS $\geq 9$ & & DEP & \\
\hline & $\mathrm{n}$ & $\%$ & $p$ & $\%$ & $p$ \\
\hline Subjects & 176 & 34 & & 24 & \\
\hline Gender & & & 0.22 & & 0.50 \\
\hline Male & 102 & 30 & & 23 & \\
\hline Female & 74 & 39 & & 27 & \\
\hline Age years & & & 0.22 & & 0.72 \\
\hline$<70$ & 56 & 43 & & 27 & \\
\hline $70-80$ & 79 & 30 & & 22 & \\
\hline$>80$ & 41 & 29 & & 27 & \\
\hline Place of domicile & & & 0.87 & & 0.76 \\
\hline Tampere & 69 & 33 & & 23 & \\
\hline another municipality & 107 & 35 & & 25 & \\
\hline Use of walking aids & & & 0.06 & & 0.049 \\
\hline yes & 100 & 40 & & 30 & \\
\hline no & 76 & 26 & & 17 & \\
\hline Smoking history in pack-years & & & 0.008 & & 0.02 \\
\hline No smoking history & 50 & 28 & & 24 & \\
\hline$<20$ & 22 & 19 & & 9 & \\
\hline 20-40 & 55 & 29 & & 18 & \\
\hline$>40$ & 49 & 53 & & 39 & \\
\hline Device treatment & & & 0.16 & & 0.13 \\
\hline oxygen therapy & 65 & 27 & & 31 & \\
\hline non-invasive ventilation & 70 & 30 & & 19 & \\
\hline both & 19 & 42 & & 37 & \\
\hline none & 22 & 18 & & 14 & \\
\hline $\mathrm{BMI}+\mathrm{kg} / \mathrm{m}^{2}$ & & & 0.65 & & 0.57 \\
\hline underweight $<18.5$ & 11 & 18 & & 18 & \\
\hline normal 18.5-24.9 & 50 & 38 & & 30 & \\
\hline overweight 25-29.9 & 39 & 36 & & 26 & \\
\hline obese $\geq 30$ & 72 & 32 & & 19 & \\
\hline Chronic obstructive pulmonary disease & & & 0.12 & & 0.51 \\
\hline yes & 103 & 39 & & 26 & \\
\hline no & 73 & 27 & & 22 & \\
\hline Obstructive sleep apnoea & & & 0.52 & & 0.62 \\
\hline yes & 56 & 38 & & 27 & \\
\hline no & 120 & 33 & & 23 & \\
\hline Obesity hypoventilation & & & 0.44 & & 0.50 \\
\hline yes & 35 & 29 & & 20 & \\
\hline no & 141 & 36 & & 26 & \\
\hline History of depression & & & 0.002 & & 0.01 \\
\hline yes & 31 & 58 & & 42 & \\
\hline no or not known & 145 & 29 & & 21 & \\
\hline
\end{tabular}

†BMI = Body mass index 
Table 3. The associations of DEPS scores with lung function parameters, walking test distance, and assessment tests in two groups with different cut-off points (DEPS $<9$ vs $\geq 9$ and DEPS $<12$ vs $\geq 12$ ).

\begin{tabular}{|c|c|c|c|c|c|c|c|c|}
\hline & \multirow[b]{2}{*}{$\mathrm{n}$} & \multirow[b]{2}{*}{ All patients ${ }^{\ddagger}$} & \multicolumn{3}{|l|}{ DEPS } & \multicolumn{3}{|l|}{ DEPS } \\
\hline & & & $<9$ & $\geq 9$ & $p$ & $<12$ & $\geq 12$ & $p$ \\
\hline Microspirometry $\mathrm{FEV}_{1}$ & 101 & & & & & & & \\
\hline$\%$ predicted $\dagger$ & & $40(12-91)$ & $42(12-91)$ & 39 (17-79) & 0.65 & $43(12-91)$ & $36(17-79)$ & 0.16 \\
\hline Spirometry $^{1} \mathrm{FEV}_{1}$ & 88 & & & & 1.0 & & & 0.9 \\
\hline$\%$ predicted ${ }^{\dagger}$ & & $55(19-114)$ & 55 (19-95) & $52(25-114)$ & & $55(19-104)$ & $51(25-114)$ & \\
\hline litres' ${ }^{\dagger}$ & & $1.4(0.4-3.6)$ & $1.5(0.4-3.3)$ & $1.4(0.7-3.6)$ & & $1.5(0.4-3.3)$ & $1.5(0.7-3.6)$ & \\
\hline CAT $^{2,3,4}$ score $^{\dagger}$ & 92 & $22(2-36)$ & $19(2-33)$ & $25(11-36)$ & 0.001 & $19(2-36)$ & $25(16-33)$ & 0.001 \\
\hline $\mathrm{mMRC}^{2,3,4}$ score $^{\dagger}$ & 95 & $3(1-4)$ & $3(1-4)$ & $3.5(2-4)$ & 0.001 & $3(1-4)$ & $3.5(3-4)$ & 0.001 \\
\hline AUDIT-C $C^{5}$ score $^{\dagger}$ & 132 & $1(0-12)$ & $0(0-10)$ & $1(0-12)$ & 0.04 & $1(0-10)$ & $1(0-12)$ & 0.62 \\
\hline
\end{tabular}

†All numbers include median (range)

‡The patients who filled the DEPS.

1 Performed within two years prior to the visit.

2 The COPD Assessment Test (CAT) and the modified MRC Dyspnoea Test were performed only by the COPD patients.

${ }^{3}$ CAT scores can range from 0 to 40 . Scores $>20$ mean that the patient's symptoms of lung disease have a high impact on the patient's perceived health status.

${ }^{4}$ Scores on the modified MRC Dyspnoea Test can range from 0 to 4 , with a score of 4 indicating that the patient is too breathless to leave the house or becomes breathless when dressing or undressing.

${ }^{5}$ AUDIT-C, the three first questions of the Alcohol Use Disorders Identification Test scores range from 0 to 12 , with a score 0 indicating no alcohol use and a score $>5$ (among women) and $>6$ (among men) indicating risky drinking that may cause health problems. 